\title{
Electrical behaviour of lanthanum- and cobalt-doped strontium stannate
}

\author{
OM PARKASH, K D MANDAL, C C CHRISTOPHER, M S SASTRY \\ and DEVENDRA KUMAR* \\ School of Materials Science and Technology, *Department of Ceramic Engineering, Institute \\ of Technology, Banaras Hindu University, Varanasi 221005 , India
}

MS received 12 April 1993; revised 4 April 1994

\begin{abstract}
Electrical properties of lanthanum- and cobalt-doped strontium stannate, $\mathrm{SrSnO}_{3}$, have been studied as a function of temperature. All the compositions investigated have cubic structure. Measurement of Seebeck coefficient in one of the cobalt-doped $\mathrm{SrSnO}_{3}$ sample shows that it exhibits p-type conductivity. Results of AC conductivity measurements show that conduction occurs by hopping of charge carriers in lanthanum-doped samples and for $x=0.01$ in cobalt-doped sample. In $x=0.05$ sample in cobalt-doped system, conduction seems to occur in extended states.
\end{abstract}

Keywords. Strontium stannate; electrical properties.

\section{Introduction}

Titanates, stannates, zirconates, etc. with the general formula $\mathrm{ABO}_{3}(\mathrm{~A}=\mathrm{Ca}, \mathrm{Sr}, \mathrm{Ba}$, $\mathrm{Pb} ; \mathbf{B}=\mathrm{Ti}, \mathrm{Sn}, \mathrm{Zr}$ ) having perovskite structure are useful materials as they find applications in a wide variety of electronic devices (Jaffe et al 1971; Lines and Glass 1977; Goodman 1986). Undoped and lanthanum-doped $\mathrm{SrSnO}_{3}$ is used as humidity sensor (Shimizu et al 1989). It has been reported that substitution of $\mathrm{La}^{3+}$ in $\mathrm{Ba}^{2+}$ site in $\mathrm{BaTiO}_{3}$ or $\mathrm{Sr}^{2+}$ site in $\mathrm{SrTiO}_{3}$ is compensated by vacancies in $\mathrm{Ba}^{2+}$ or $\mathrm{Sr}^{2+}$ sublattice respectively for substitutions of more than $1 \%$ (Newnham 1983). This is represented by the formula $\mathrm{M}_{1-3 x / 2} \mathrm{La}_{x}\left(V_{\mathrm{M}}^{\prime \prime}\right)_{x / 2} \mathrm{TiO}_{3}\left(\mathrm{M}=\mathrm{Ba}^{2+}\right.$ or $\left.\mathrm{Sr}^{2+}\right)$. Similarly substitution of $\mathrm{Co}^{3+}$ in $\mathrm{Ti}^{4+}$ site is compensated by creation of vacancies in oxygen sublattice as represented by the formula $\mathrm{MTi}_{1-x} \mathrm{Co}_{x} \mathrm{O}_{3-x / 2}\left(\mathrm{M}=\mathrm{Ba}^{2+}\right.$ or $\left.\mathrm{Sr}^{2+}\right)$. In this paper we report the electrical behaviour of a few compositions of lanthanum- and cobalt-doped strontium stannate prepared by ceramic method.

\section{Experimental}

Lanthanum- and cobalt-doped strontium stannate samples were prepared by ceramic method using strontium carbonate, lanthanum oxalate, tin(IV) oxide and cobalt oxalate, all having purity better than $99.5 \%$. Lanthanum oxalate was estimated as lanthanum oxide. Appropriate quantities of these materials were accurately weighed, mixed and ground under acetone in an agate mortar for $6 \mathrm{~h}$. The dried powders were transferred to platinum crucibles and calcined at $1473 \mathrm{~K}$ for $6 \mathrm{~h}$ in air and then cooled to room temperature. The calcined powders were ground, mixed with a few drops of $1 \%$ solution of polyvinyl alcohol (PVA) as binder, and pressed as cylindrical pellets. These pellets were slowly heated to $675 \mathrm{~K}$ and kept at this temperature for $1 \mathrm{~h}$ for complete removal of the binder. The temperature was then raised to $1623 \mathrm{~K}$ and the samples were sintered in air at this temperature for $12 \mathrm{~h}$ and then cooled slowly.

$\mathrm{X}$-ray diffraction patterns of the crushed pellets were taken using $\mathrm{Cu}-\mathrm{K}_{\alpha}$ radiation 
in a Rigaku Rotaflex diffractometer. DC resistance was measured as a function of temperature using a Keithley digital electrometer model 616 by two-probe technique. Four-probe technique was used for composition with $x=0.05$ in the $\operatorname{SrSn}_{1-x} \mathrm{Co}_{x} \mathrm{O}_{3}$ system as it had low resistance. Seebeck coefficient $(\alpha)$ was measured on thick sintered cylindrical pellet relative to platinum. AC conductance $(G)$ and dielectric loss $(D)$ were measured as a function of frequency $(f)$ at $350 \mathrm{~K}$ using a Hewlett Packard 4192A LF impedance analyser.

\section{Results and discussion}

Compositions with $x=0.01$ and 0.05 in the systems $\mathrm{Sr}_{1-3 x / 2} \mathrm{La}_{x} \mathrm{SnO}_{3}$ and $\mathrm{SrSn}_{1-x}$ $\mathrm{Co}_{x} \mathrm{O}_{3}$ were synthesized. Powder X-ray diffraction patterns of the various compositions indicate the formation of single-phase solid solution in each case. X-ray data of all the samples could be indexed on the basis of a cubic unit cell similar to $\mathrm{SrSnO}_{3}$ (Parkash et al 1994). The values of lattice parameter $a$ for all the compositions are given in table 1 . It is observed that $a$ decreases with increasing $x$ in both the systems. This can be understood in terms of ionic radii of $\mathrm{Sr}^{2+}(1.44 \AA), \mathrm{La}^{3+}(1.32 \AA)$, $\mathrm{Sn}^{4+}(0.69 \AA)$ and $\mathrm{Co}^{3+}(0.61 \AA$ or $0.525 \AA$ depending on whether it exists in the high-spin state $t_{2 g}^{4} e_{g}^{2}$ or low-spin state $t_{2 g}^{6} e_{g}^{0}$ ) (Shannon and Prewitt 1969).

Variation of Seebeck coefficient $\alpha$ with temperature $\mathrm{K}$ of the sample $x=0.05$ in the $\mathrm{SrSn}_{1-x} \mathrm{Co}_{x} \mathrm{O}_{3}$ system is shown in figure 1. The value of $\alpha$ is found to be positive

Table 1. Lattice parameter (a), activation energy $\left(E_{a}\right)$ for $\mathrm{DC}$ conductivity and $s$ values for samples in $\mathrm{SrSn}_{1-x} \mathrm{Co}_{x} \mathrm{O}_{3}$ and $\mathrm{Sr}_{1-3 x / 2} \mathrm{La}_{x} \mathrm{SnO}_{3}$ systems.

\begin{tabular}{lcccc}
\hline System & $x$ & $a(\AA)$ & $\begin{array}{c}\text { Activation } \\
\text { energy (eV) }\end{array}$ & $\begin{array}{c}s \\
(350 \mathrm{~K})\end{array}$ \\
\hline $\mathrm{Sr}_{1-3 x / 2} \mathrm{La}_{x} \mathrm{SnO}_{3}$ & 0.01 & 4.026 & 1.01 & 0.70 \\
$\mathrm{SrSn}_{1-x} \mathrm{Co}_{x} \mathrm{O}_{3}$ & 0.05 & 4.023 & 1.07 & 0.80 \\
& 0.01 & 4.023 & 0.54 & 0.80 \\
& 0.05 & 4.017 & 0.23 & - \\
\hline
\end{tabular}

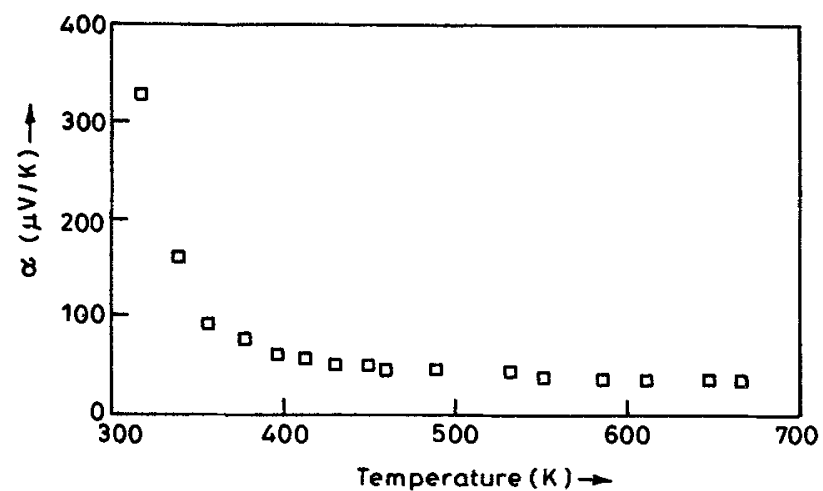

Figure 1. Variation of Seebeck coefficient $\alpha$ with temperature in the sample $\mathrm{SrSn}_{0.95} \mathrm{Co}_{0.05} \mathrm{O}_{3}$. 
over the entire temperature range of measurement. This shows that holes are the majority charge carriers. It is observed from figure 1 that $\alpha$ decreases sharply in the temperature range $300-400 \mathrm{~K}$. Thereafter, it remains almost independent of temperature up to $700 \mathrm{~K}$. The $p$-type behaviour of this composition may be due to generation of holes to compensate for the deficiency of one positive charge in $\mathrm{Co}^{3+}$ compared to $\mathrm{Sn}^{4+}$. Measurement of Seebeck coefficient of other samples was not possible due to their high resistance.

Plots of logarithm of $\mathrm{DC}$ resistivity $\left(\log \rho_{\mathrm{dc}}\right)$ vs $1000 / T$ are shown in figures 2 and 3 . The data in figures 2 and 3 were fitted by least-squares method to a straight line and the values of activation energy $E_{a}$ calculated from the slopes of these lines
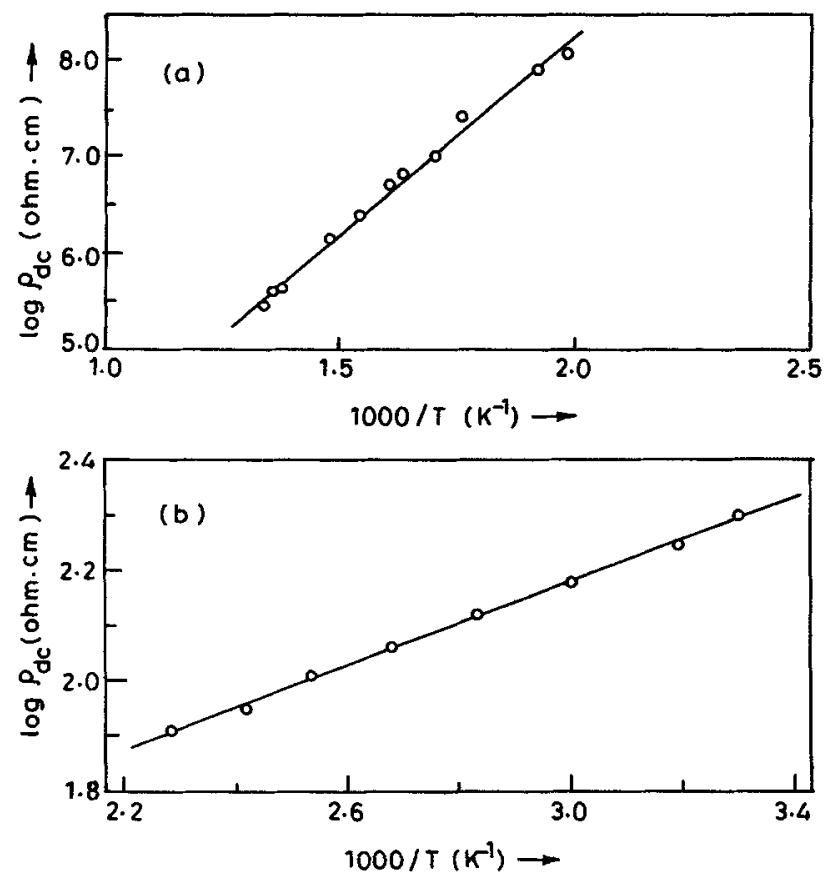

Figure 2. Plots of $\log \rho_{\mathrm{dc}}$ vs $1000 / T\left(\mathrm{~K}^{-1}\right)$ for (a) $\mathrm{SrSn}_{0.99} \mathrm{Co}_{0.01} \mathrm{O}_{3}$ and (b) $\mathrm{SrSn}_{0.95}$ $\mathrm{Co}_{0.05} \mathrm{O}_{3}$.

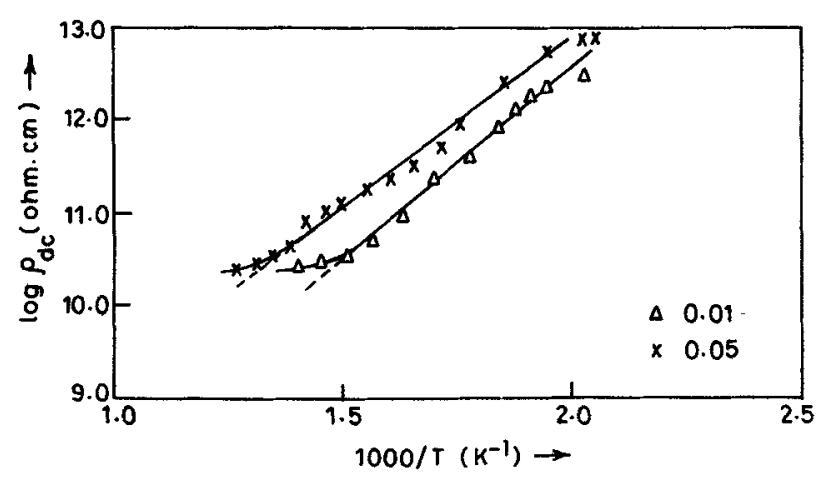

Figure 3. Plot of $\log \rho_{\mathrm{dc}}$ vs $1000 / T\left(\mathrm{~K}^{-1}\right)$ for $\mathrm{Sr}_{1-3 x / 2} \mathrm{La}_{x} \mathrm{SnO}_{3}$. 
are given in table 1 . The values of activation energy for conduction for the compositions with $x=0.01$ and 0.05 in the $\mathrm{Sr}_{1-3 x / 2} \mathrm{La}_{x} \mathrm{SnO}_{3}$ system are almost equal $(\sim 1.00 \mathrm{eV})$. This shows that the conduction mechanism is the same in both samples. The samples of $\mathrm{SrSn}_{1-x} \mathrm{Co}_{x} \mathrm{O}_{3}$ system have different $E_{a}$ values, much less than those of the lanthanum-doped samples.

The much lower resistivity in the cobalt-doped samples compared to lanthanumdoped samples is due to the difference in the mechanism of compensation in the two cases. In the case of lanthanum-doped strontium stannate, charge compensation occurs due to creation of vacancies in $\mathrm{Sr}^{2+}$ sublattice:

$$
\mathrm{La}_{2} \mathrm{O}_{3} \stackrel{3 \mathrm{SrO}}{\longrightarrow} 2 \mathrm{La}_{\mathrm{Sr}}+\mathrm{V}_{\mathrm{Sr}}^{\prime \prime}+3 \mathrm{O}_{\mathrm{O}}
$$

These strontium ion vacancies do not contribute significantly to the conductivity of these materials because movement of large $\mathrm{Sr}^{2+}$ or $\mathrm{La}^{3+}$ ions in close-packed oxygen ions is very difficult. On the other hand $\mathrm{La}^{3+}$ ions act as impurity scattering centres. This explains the higher resistivity of the samples with $x=0.05$ compared to $x=0.01$ in the lanthanum-doped system. On substituting trivalent cobalt in tetravalent tin sites, charge compensation occurs through generation of holes:

$$
2 \mathrm{Co}^{3+} \stackrel{2 \mathrm{Sn}^{4+}}{\longrightarrow} 2 \mathrm{Co}_{\mathrm{Sn}}^{\prime}+2 \mathrm{~h} .
$$

The charge compensation is therefore electronic in nature. This accounts for the drastic decrease in resistivity with cobalt substitution. This is in contrast to the ionic compensation which occurs in lanthanum-doped samples.

In order to understand the mechanism of conduction, $\mathrm{AC}$ conductivity was measured as a function of frequency in the range $1 \mathrm{kHz}-1 \mathrm{MHz}$ at $350 \mathrm{~K}$ in the cobalt- $(x=0.05)$ and lanthanum-doped samples. Plots of $\log \sigma_{\mathrm{ac}}$ vs $\log f$ are shown in figure 4 . It is noted that $\mathrm{AC}$ conductivity obeys the relation

$$
\sigma_{\mathrm{ac}}=A w^{\mathrm{s}},
$$

for both the lanthanum-doped samples. Values of $s$ found by least-squares fitting of data are given in table 1. Frequency dependence of AC conductivity in these samples

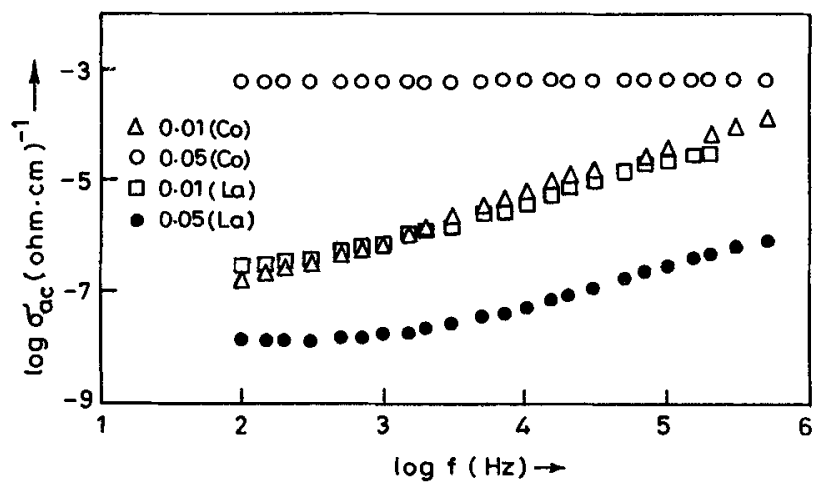

Figure 4. Variation in $\log \sigma_{\text {ac }}$ as a function of frequency at $350 \mathrm{~K}$ for $\mathrm{SrSn}_{1-x} \mathrm{Co}_{x} \mathrm{O}_{3}$ and $\mathrm{Sr}_{1-3 \times / 2} \mathrm{La}_{x} \mathrm{SnO}_{3}$ systems. 
shows that conduction occurs by hopping of charge carriers among localized sites (Mott and Davis 1979). In lanthanum-doped system these localized sites are associated with $\mathrm{Sn}^{2+}$ and $\mathrm{Sn}^{4+}$ ions. Traces of divalent $\mathrm{Sn}^{2+}$ ions may be present because of loss of oxygen occurring during sintering of these materials at high temperatures in accordance with the reaction (Burn and Neirman 1982)

$$
\mathrm{O}_{\mathrm{o}} \rightarrow 1 / 2 \mathrm{O}_{2}+\mathrm{V}_{\mathrm{o}}+2 e^{\prime}
$$

where all the species are written in accordance with Kröger-Vink notation of defects. The electrons released in the above reaction may be captured by $\mathrm{Sn}^{4+}$ to generate $\mathrm{Sn}^{2+}$. Conduction therefore seems to occur by hopping of charge carriers among $\mathrm{Sn}^{4+}$ and $\mathrm{Sn}^{2+}$ sites in lanthanum-doped samples. In cobalt-doped samples $\mathrm{Co}^{3+}$ ions seems to give rise to localized states between the valence band and conduction band. A slight amount of $\mathrm{Co}^{2+}$ may be generated due to capture of electrons released in reaction (4). Conduction then seems to occur by excitation of charge carriers at valence band edge and hopping at energies close to it. This mechanism is similar to that found in valence-compensated systems $\mathrm{Ba}_{1-x} \mathrm{La}_{x} \mathrm{Ti}_{1-x} \mathrm{Co}_{x} \mathrm{O}_{3}, \mathrm{Ca}_{1-x} \mathrm{La}_{x} \mathrm{Ti}_{1-x} \mathrm{Co}_{x} \mathrm{O}_{3}$ and $\mathrm{Ca}_{1-x} \mathrm{Y}_{x} \mathrm{Ti}_{1-x} \mathrm{Co}_{x} \mathrm{O}_{3}$ (Kumar et al 1990; Parkash et al 1990; Parkash et al 1993). With increasing concentration of cobalt ions in the $\mathrm{SrSn}_{1-x} \mathrm{Co}_{x} \mathrm{O}_{3}$ system, the acceptor levels associated with $\mathrm{Co}^{3+}$ seem to move closer to the valence band. In the sample with $x=0.05$, conduction seems to occur by excitation of holes in the valence band. This is confirmed by frequency-independent behaviour of AC conductivity. If conduction occurs in extended states, then the AC conductivity should remain independent of frequency up to $10^{7} \mathrm{~Hz}$ (Mott and Davis 1979). This is found to be so in the samples with $x=0.05$ in cobalt-doped samples. Further, the very low value of activation energy $E_{a}$ in this sample is in conformity with this mechanism.

\section{Acknowledgements}

The authors are thankful to the Department of Science and Technology, New Delhi, for financial assistance. One of the authors (KDM) is thankful to CSIR, New Delhi, for Research Associateship.

\section{References}

Burn I and Neirman S 1982 J. Mater. Sci. 173510

Devendra Kumar, Prasad Ch. D and Parkash O 1990 J. Phys. Chem. Solids 5173

Goodman G 1986 in Ceramic materials for electronic applications (ed.) R C Buchanan (New York: Marcel Dekker) p. 79

Jaffe B, Cook Jr W K and Jaffe H 1971 Piezoelectric ceramics (London/New York: Academic Press) Ch. 5 and 12

Lines M E and Glass A M 1977 Principle and applications of ferroelectric and related materials (Oxford: Clarendon Press) Ch 12

Mott NF and Davis E A 1979 Electronic processes in non crystalline solids (Oxford: Clarendon Press) II Ed

Newnham R E 1983 J. Mater. Educ. 5941

Parkash O, Devendra Kumar, Prasad Ch. D and Tewari H S 1990 J. Phys. D. Appl. Phys. 23342

Parkash O, Tewari H S, Tare V B and Devendra Kumar 1993 J. Phys. D. Appl. Phys. 26676

Parkash O, Mandal K D, Christopher C C, Mandalika S Sastry and Devendra Kumar 1994 J. Mater. Sci. Lett. (Communicated)

Shimizu Y, Shimalukuro M, Arai H and Seiyama T 1989 J. Electrochem. Soc. 1361206

Shannon R D and Prewitt C T 1969 Acta Crystallogr. B25 925 